\title{
Brote de infección nosocomial por Serratia marcescens asociado a contaminación intrínseca de clorhexidina acuosa
}

\author{
Beatrice Hervé, May Chomali, Cecilia Gutiérrez, Mariana Luna, Jeannette Rivas, Rodrigo Blamey, \\ Ricardo Espinoza, Giannina Izquierdo, Catalina Cabezas, Claudia Álvarez y Sebastián de la Fuente
}

\section{Outbreak due to Serratia marcescens associated with intrinsic contamination of aqueous chlorhexidine}

Serratia marcescens is a widely distributed gram-negative rod, often associated to nosocomial infections. Some outbreaks linked to contaminated antiseptic solutions have been reported. In this study we report a nosocomial outbreak of surgical site infection and catheter insertion site infection due to $S$. marcescens. 33 patients with positive cultures were studied after an index case was identified. Epidemiological, microbiological and molecular analysis demostrated an intrinsic contamination of alcohol free chlorhexidine solution as causal factor. Positive cultures were associated with 13 clinical infections, 9 colonized patients, 6 pseudobacteremia episodes and 5 patients without documented exposure. Hospital and national recall of contaminated chlorhexidine solution was performed after this study. Intrinsic contamination of antiseptic solutions is an infrequent cause of nosocomial infections with major epidemiological relevance.

Key words: Serratia marcescens, nosocomial outbreak, intrinsic contamination, chlorhexidine.

Palabras clave: Serratia marcescens, brote nosocomial, contaminación intrínseca, clorhexidina.

\section{Introducción}

S erratia marcescens es un bacilo gramnegativo de la Familia Enterobacteriaceae, de amplia distribución en el ambiente (agua, tierra) y microbiota comensal de humanos y animales ${ }^{1,2}$. Se ha descrito a $S$. marcescens como agente causal de múltiples infecciones nosocomiales, entre las que destacan brotes de infección urinaria, sepsis e infección de sitio operatorio (ISO) en Unidades Críticas de Adultos y Neonatales ${ }^{3-8}$, e ISO, LCR, catéteres endovenosos, asociadas a contaminación extrínseca de diversos antisépticos ${ }^{9-13}$.

Además existen infecciones esporádicas o endémicas por $S$. marcescens, que en nuestra institución alcanzaron un promedio de 1,5 casos por mes o 0,35 casos por semana durante el año 2013. Estas infecciones esporádicas son en su mayoría de foco respiratorio o urinario, en pacientes ambulatorios o que ingresan al hospital por cuadros adquiridos en la comunidad, lo que está descrito en la literatura médica ${ }^{2}$.

Se han descrito brotes de infección asociados al uso de antisépticos, por contaminación extrínseca del antiséptico (es decir, al manipularlo el usuario lo contamina), o por contaminación intrínseca (el antiséptico está contaminado de fábrica), para los distintos tipos de antisépticos, debido a diferentes microorganismos, generalmente bacilos gramnegativos. Sin embargo, hasta la fecha no ha sido descrita la contaminación intrínseca de clorhexidina por $S$. marcescens ${ }^{10}$.

En el presente trabajo se reporta la ocurrencia de un brote de ISO y de infección de sitio de inserción de catéter (SIC) por $S$. marcescens asociado a la contaminación intrínseca de clorhexidina acuosa tinturada.

\section{Método}

\section{Antecedentes}

El 25 de octubre de 2014 se obtiene cultivo positivo para $S$. marcescens en la secreción de herida operatoria en una paciente con implante mamario bilateral, al quinto día post operatorio, con diagnóstico clínico de infección de sitio quirúrgico.

El 26 de octubre de 2014 se obtuvo un segundo cultivo positivo para $S$. marcescens en hemocultivo de un paciente sometido a neurocirugía 9 días antes, que presentó filtración de LCR por la herida quirúrgica, la cual se re-suturó y evolucionó con fiebre.

Dado que se trataba de un aumento respecto de nuestros indicadores previos (la tasa de ISO por implante mamario en la institución había sido 0 x 100 cirugías los últimos 3 años y el $\mathrm{n}^{\circ}$ de cultivos positivos a $S$. marcescens por semana los últimos 20 meses había sido de 0,3), se consideró un brote y se dio inicio inmediatamente a una investigación epidemiológica para definir factores de riesgo y posible fuente de la infección.
Clínica Las Condes, Santiago, Chile.

Comité de IAAS y Laboratorio de Microbiología.

Los autores declaran no tener conflictos de interés en este trabajo. Trabajo realizado sin fuente de financiamiento externo.

Recibido: 27 de febrero de 2015 Aceptado: 10 de julio de 2015

Correspondencia a: Beatrice Hervé Espejo bherve@clc.cl 


\section{Investigación inicial y definiciones}

En una primera etapa se incluyó en el estudio todo cultivo positivo a $S$. marcescens obtenido en el laboratorio de microbiología durante el mes previo al caso índice. Posteriormente, y de acuerdo al análisis de registros y categorización según evolución clínica, se ajustó la definición de caso a ISO o SIC con cultivo positivo a $S$. marcescens, cuya cirugía, instalación de catéter o curación de catéter hubiera ocurrido entre el 25 de septiembre y 7 de noviembre de 2014, (tomando en cuenta como fechas un mes previo a la detección del caso índice, hasta el día en que se detectó el producto y lote contaminado y se retirara de circulación). En base a los hallazgos se definieron tres categorías de pacientes:

- Infectado: Aquel paciente con cultivo positivo a $S$. marcescens, que presentó repercusión clínica en su evolución (parámetros inflamatorios alterados) y que requirió tratamiento antimicrobiano y/o reintervención quirúrgica en el caso de ISO o retiro de catéter por ese cultivo, en caso de ISC.

- Colonizado: Aquellos pacientes con cultivo positivo a S. marcescens, que no presentaron repercusión clínica ni alteración de parámetros inflamatorios en relación al cultivo, y que no requirieron terapia antimicrobiana.

- Paciente con pseudo-bacteriemia: Aquellos pacientes que presentaron hemocultivo positivo a $S$. marcescens sin repercusión clínica y no requirieron tratamiento antimicrobiano por este microorganismo.

Para cada cultivo positivo a $S$. marcescens encontrado hasta un mes previo al caso índice, se determinó: fecha de obtención, tipo de muestra, servicio de procedencia y antibiotipo, y se revisó registros en su ficha clínica buscando: procedimientos invasores, personal a cargo, personal de toma de muestra, e insumos utilizados. También se revisó los registros de evolución clínica, para definir si el cultivo positivo correspondió a infección, a pseudo-bacteriemia o colonización, según las definiciones establecidas.

\section{Estudio epidemiológico}

Se puede sistematizar en diferentes etapas:

- Debido a que se encontró numerosos casos con cultivo positivo de ingreso a Clínica Las Condes, se consultó con otros centros la existencia de situación similar, con el fin de descartar que se estuviera en presencia de un brote comunitario.

- Dado que hubo cultivos positivos sin repercusión clínica, se decidió hacer estudio de clonalidad, enviando las dos cepas de los casos índice, dos de pseudo-bacteriemia y una de caso catalogado como colonizado, al Laboratorio de Microbiología de la Pontificia Universidad Católica de Chile (PUC), para estudio mediante electroforesis de campo pulsado (EFCP).
- Se buscó factores comunes entre los pacientes con cultivo positivo: pabellón, médico tratante, personal de salud involucrado en atención del paciente, procedimientos invasores, etc. y se realizó entrevistas al personal involucrado en los procedimientos invasores, toma de muestras de pacientes con cultivos positivos, con el fin de detectar posibles quiebres en la técnica de preparación de piel y/o punción.

Una vez descartados los quiebres en los procedimientos, el único factor común detectado fue el uso de antiséptico en la preparación de piel (clorhexidina acuosa tinturada, al 2\%) para cirugía, toma de hemocultivos y curación de catéter, así como el uso de esponjas con clorhexidina (4\%), por lo que se decidió cultivar muestras de los lotes en uso de este antiséptico en la institución, así como de las esponjas. Cabe destacar que debido a normativa interna que exige uso único de estos insumos, sólo se recuperó frascos cerrados en su envase original en cada servicio involucrado.

\section{Estudio microbiológico}

Se procesó bajo campana de bioseguridad, cada frasco de antiséptico (ya fuera jabón de clorhexidina o clorhexidina en base acuosa tinturada) o esponja, recuperados de los servicios donde fueron atendidos los casos y que estuvieran sin abrir (sellados), según el siguiente protocolo:

- Al abrir frasco o botella, limpiar parte superior con alcohol $70^{\circ}$.

- Se obtuvo con jeringa de $1 \mathrm{ml}, 300 \mu \mathrm{l}$ e inoculó en tubos con $3 \mathrm{ml}$ de caldo cerebro/corazón (BHI).

- Se sembró directamente $100 \mu l$ en placa de agar sangre y $100 \mu 1$ en placa de agar MacConkey, diseminando con técnica de rastrillo.

- Se incubó tubo y placas a $35^{\circ} \mathrm{C}$.

- Se observó las placas por $48 \mathrm{~h}$.

- Se realizó identificación por metodología de espectometría de masas (Vitek MS), toda colonia obtenida, con recuento.

- Si el cultivo de placas fue negativo, se subcultivó $100 \mu \mathrm{l}$ de caldo a las $48 \mathrm{~h}$, en agar sangre.

- Cada frasco, una vez obtenida la muestra para siembra, se mantuvo cerrado con su tapa a $4^{\circ} \mathrm{C}$, debidamente rotulado.

- En el caso de esponjas con clorhexidina impregnada, se cortó un trozo con tijera estéril e introdujo en caldo BHI. Se incubó por $48 \mathrm{~h}$ a $35^{\circ} \mathrm{C}$ y luego se subcultivó el caldo en agar sangre y MacConkey.

Simultáneamente y para descartar contaminación dentro del laboratorio, se incubó en condiciones estándares el $5 \%$ de los frascos de hemocultivo sin inocular disponibles en ese momento (para analizar las bacteriemias), y tubos con $3 \mathrm{ml}$ de caldo BHI sin inocular. 
Posteriormente al hallazgo, se envió contra muestras del lote contaminado (frascos cerrados en su envase original), a los Laboratorios de Microbiología de la Pontificia Universidad Católica de Chile y Universidad de Chile, para su cultivo.

\section{Seguimiento}

Con posterioridad a la detección de la fuente de $S$. marcescens, se estableció un plan a seguir, consistente en el retiro de todos los productos del fabricante dentro de la institución, se comunicó al proveedor la situación y se realizó la denuncia correspondiente a la autoridad sanitaria. Se envió muestras del mismo lote involucrado a centro de referencia nacional del Instituto de Salud Pública (ISP) y se realizó vigilancia activa mediante revisión de registros de cirugías hasta tres semanas previo a la fecha de cultivo positivo de antiséptico (basado en los períodos de incubación de los casos estudiados), estableciendo un listado de pacientes en "mayor riesgo" (implantes, cirugía traumatológica), y comunicando directamente a cada cirujano los resultados de la investigación, para controlar sus pacientes en riesgo y notificar cualquier hallazgo. También se contactó a cada paciente ya infectado, explicando los hallazgos y se realizó un total de tres estudios de EFCP:

- Cepas de casos índice y pseudo-bacteriemia y muestra colonizada, en el Laboratorio de Microbiología de la PUC.

- Cepas de casos índice y cepas aisladas del antiséptico, en el Laboratorio de Microbiología de la PUC.

- Cepas de casos de infección, detectados pre y post hallazgo de $S$. marcescens en el antiséptico, realizado en el ISP.

\section{Resultados}

Nuestra investigación fue gatillada por la aparición de dos casos de infección comprobada por $S$. marcescens durante la misma semana. Muy luego en el curso de la investigación, se detectó que desde al menos 30 días previos, había un aumento en el número de cultivos positivos a $S$. marcescens, respecto de los números habituales.

En la Figura 1 se muestra la endemia mensual de $S$. marcescens durante 2013 y 2014, evidenciando un aumento de cultivos positivos a partir del mes de septiembre de 2014. En promedio, durante 2013 hubo 0,35 cultivos positivos por semana (oscilando entre 0 y 3 por mes) y durante 2014 hubo 0,96 cultivos positivos por semana (oscilando entre 1 y 12 por mes).

En la Tabla 1 se muestra los resultados de cultivos realizados a frascos de clorhexidina $2 \%$ cerrados y a esponjas, diferenciando por lote y servicio de obtención. En la Tabla 2, se muestran los cultivos positivos de- tectados durante la investigación, en que se pudo tener acceso completo a los registros clínicos, tipo de muestra y porcentaje que correspondieron a infección, a pseudobacteriemia y a colonización, según las definiciones ya descritas. Del análisis de estos 33 cultivos positivos, obtenidos antes y después del hallazgo de la fuente de $S$. marcescens, se logró definir que $6(18 \%)$ correspondieron a pseudo-bacteriemia, 9 (27\%) a colonización y 13 (42\%) a infección propiamente tal. En el período estudiado ( $25 \mathrm{de}$ septiembre a 31 de diciembre de 2014) hubo cinco cultivos positivos $(15 \%)$ en que no se pudo establecer un contacto directo con clorhexidina, y correspondieron a muestra

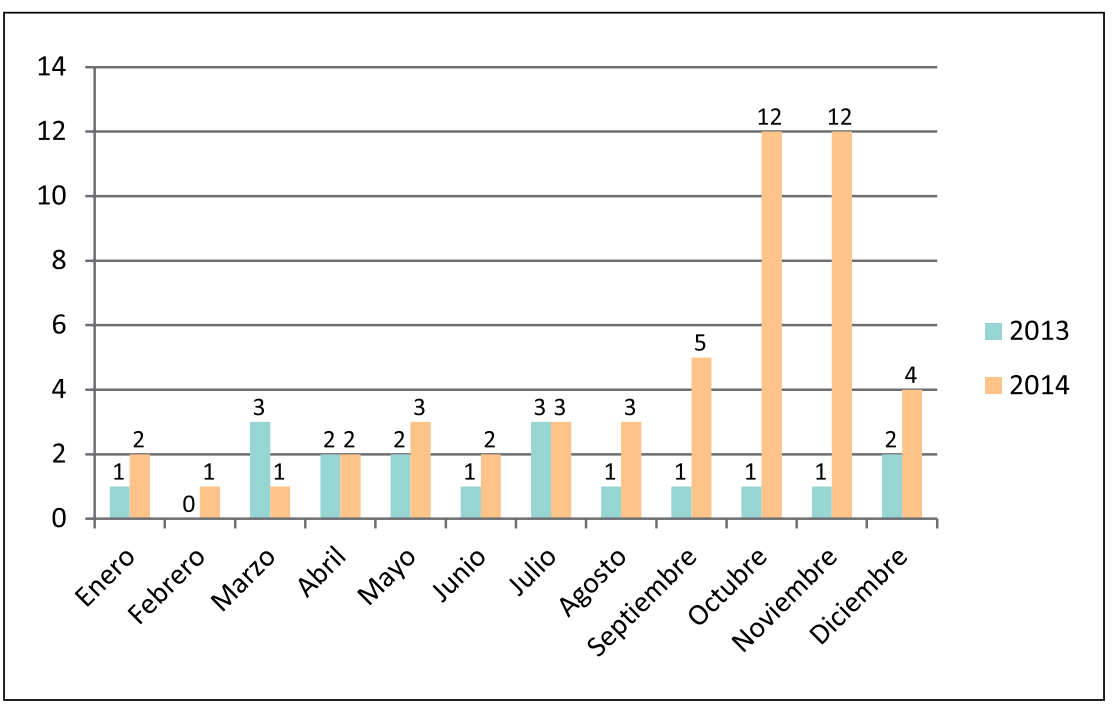

Figura 1. Distribución de casos de S. marcescens por mes.

Tabla 1. Resultado de Cultivos realizados por servicio y lotes

\begin{tabular}{|c|c|c|c|}
\hline Servicio de procedencia & Producto & Lote* & Resultado cultivo \\
\hline Pabellón & Chxd jabón & A & Negativo 96 h \\
\hline Pabellón & Chxd solución acuosa color & B & Serratia marcescens $24 \mathrm{~h}$ \\
\hline Pabellón & Esponja & $s / n$ & Negativo 96 h \\
\hline Pabellón & Chxd jabón & C & Caldo turbio $96 \mathrm{~h}$ \\
\hline Pabellón & Chxd solución acuosa color & B & Serratia marcescens $24 \mathrm{~h}$ \\
\hline Unidad Coronaria & Esponja & $\mathrm{D}$ & Negativo $96 \mathrm{~h}$ \\
\hline Unidad Coronaria & Esponja & $E$ & Negativo $96 \mathrm{~h}$ \\
\hline Unidad Coronaria & Chxd jabón & A & Negativo $96 \mathrm{~h}$ \\
\hline Unidad Coronaria & Chxd solución acuosa color & $\mathrm{F}$ & Negativo $96 \mathrm{~h}$ \\
\hline Unidad Coronaria & Chxd solución acuosa color & B & Serratia marcescens $48 \mathrm{~h}$ \\
\hline Pediatría & Chxd jabón & G & Negativo 96 h \\
\hline Pediatría & Chxd solución acuosa color & $\mathrm{F}$ & Negativo $96 \mathrm{~h}$ \\
\hline
\end{tabular}


respiratoria y/o urinaria. En la Tabla 3 se presentan las características de las infecciones en que se pudo establecer uso de clorhexidina previo al cultivo positivo.

En total fueron 13 infecciones (6 mujeres, 7 varones), con edad promedio 46,9 años (22-84). El tiempo de incubación fue de 11,3 ds en las ISO (1-24). En las infecciones de SIC, dado que hubo múltiples exposiciones (múltiples curaciones realizadas), no se pudo definir el tiempo de incubación.

En cuanto a enfermedad de base, hubo pacientes oncológicos (n: 3), con insuficiencia renal (n: 3) y cirugía

\section{Tabla 2. Distribución 33 cultivos positivos según repercusión clínica}

\begin{tabular}{|c|c|c|c|}
\hline Tipo de cuadro clínico & n de cultivos & $\%$ & Tipo de muestra \\
\hline Colonización: sin repercusión clínica & 9 & 27 & $\begin{array}{l}5 \text { piel, } \\
4 \text { punta de catéter }\end{array}$ \\
\hline $\begin{array}{l}\text { Pseudobacteremia sin repercusión } \\
\text { clínica }\end{array}$ & 6 & 18 & 6 hemocultivo positivo de ingreso* \\
\hline Infección & 13 & 42 & $\begin{array}{l}7 \text { infección de sitio operatorio, } \\
3 \text { infección sitio inserción catéter, } \\
2 \text { ITS asociada a catéter, } \\
1 \text { ITS post quirúrgica }\end{array}$ \\
\hline $\begin{array}{l}\text { Cuadro clínico respiratorio o urinario, } \\
\text { en que no se pudo demostrar con- } \\
\text { tacto con clorhexidina }\end{array}$ & 5 & 15 & $\begin{array}{l}4 \text { secreción bronquial, } \\
1 \text { orina }\end{array}$ \\
\hline $\begin{array}{l}\text { Total cultivos positivos a S. marcescens } \\
\text { sept-dic } 2014\end{array}$ & 33 & 100 & \\
\hline
\end{tabular}

plástica o reparadora (n: 3), pacientes neuroquirúrgicos (n: 2) y un paciente politraumatizado grave. En siete de las ocho heridas infectadas, fue necesario realizar aseo quirúrgico; en dos de ellos, aseo en más de una oportunidad. En cuatro de cinco pacientes en que hubo instalación de dispositivos o implante en la cirugía inicial, éste tuvo que ser retirado.

El estudio microbiológico de antisépticos detectó tres frascos positivos a $S$. marcescens, correspondientes los tres al mismo lote. Dos de los tres frascos fueron recuperados de la bodega de Pabellón Central, el otro de la bodega de Unidad Coronaria. Los demás lotes estudiados y las esponjas cultivadas dieron resultado negativo. Todos los controles (frascos de hemocultivo y tubos con caldo BHI incubados sin inocular) dieron resultado negativo.

Paralelamente, se envió contra muestra de 10 frascos sellados del lote a los laboratorios de Microbiología de la Pontificia Universidad Católica de Chile y Universidad de Chile. Ambos laboratorios confirmaron nuestro hallazgo.

Posteriormente a la fecha del hallazgo de la fuente, y durante el período establecido de vigilancia, se encontraron nueve nuevos cultivos positivos a $S$. marcescens. Los pacientes fueron investigados según los mismos parámetros de la investigación inicial, encontrándose que todos ellos habían estado expuestos previo a la fecha de retiro del antiséptico contaminado y siete de ellos correspondieron a infección (cuatro ISO, tres infección acceso vascular) y dos a colonización.

De las EFCP realizadas, en los tres análisis se encontró que las cepas eran indistinguibles entre sí, confirmando así la característica monoclonal del brote.

\begin{tabular}{|c|c|c|c|c|c|c|c|c|}
\hline Sexo & Edad & Diagnóstico de base & Tipo de muestra & $\begin{array}{l}\text { Tipo de } \\
\text { infección }\end{array}$ & $\begin{array}{c}\text { Fecha de contacto con } \\
\text { desinfectante contaminado }\end{array}$ & $\begin{array}{l}\text { Fecha } \\
\text { de dg }\end{array}$ & $\begin{array}{l}\text { Rehospi- } \\
\text { talización }\end{array}$ & $\begin{array}{l}\text { Reinter- } \\
\text { vención }\end{array}$ \\
\hline Masc & 28 & Neuroquirúrgico & Hemocultivo & ITS & 25 -oct & 26-oct & Sí & No \\
\hline Fem & 51 & Cirugía plástica & Herida operatoria & ISO & 20-oct & 25 -oct & Sí & Sí \\
\hline Fem & 80 & Cirugía mayor de columna & Herida operatoria & ISO & 24-oct & 06-nov & Sí & Sí \\
\hline Fem & 76 & Insuficiencia renal & Hemocultivo catéter de diálisis & ITS-CVC & Múltiples en oct y nov & 07-nov & No & No \\
\hline Masc & 25 & Oncológico & Hemocultivo por arrastre CVC & ITS-CVC & Múltiples desde 26 de sept & 10-nov & No & No \\
\hline Fem & 38 & Cirugía reconstructiva & Herida operatoria & ISO & 30-oct & 13-nov & Sí & Sí \\
\hline Masc & 43 & Politraumatizado & Herida fractura expuesta & ISO & 03-nov & 10-nov & No & Sí \\
\hline Masc & 31 & Oncológico & Herida operatoria implantcfix & ISO & Múltiples desde 27-oct & 15-nov & Sí & Sí \\
\hline Masc & 84 & Insuficiencia renal & Sitio de inserción catéter diálisis & ISC & Múltiples en oct y nov & 20-nov & No & No \\
\hline Masc & 22 & Neuroquirúrgico & Herida operatoria & ISO & 03-nov & 18-nov & Sí & Sí \\
\hline Masc & 29 & Oncológico & Sitio de inserción CVC & ISC & Múltiples desde 15 -oct & 19-nov & No & No \\
\hline Fem & 83 & Insuficiencia renal & Sitio de inserción catéter diálisis & ISC & Múltiples durante octubre y nov & 28-nov & No & No \\
\hline Fem & 40 & Cirugía reconstructiva & Herida operatoria & ISO & 07-nov & $01-$ dic & Sí & Sí \\
\hline
\end{tabular}




\section{Discusión}

Describimos en el presente documento la investigación epidemiológica y microbiológica realizada a raíz de dos casos inusuales de infección nosocomial por $S$. marcescens ocurridos durante la misma semana calendario. Esta investigación nos permitió detectar la fuente del microorganismo en un lote de frascos de clorhexidina acuosa tinturada $2 \%$, contaminado de fábrica.

La clorhexidina posee un amplio espectro de acción, siendo bactericida sobre bacterias grampositivo y gramnegativo, bacteriostática sobre micobacterias. Su sitio de acción primario es la membrana citoplasmática, produciendo modificación en la permeabilidad ${ }^{14}$.

Se ha reportado que cepas de Acinetobacter baumannii, Pseudomonas aeruginosa y Klebsiella pneumoniae pueden replicarse en presencia de $1 \%$ de clorhexidina ${ }^{15}$ así como está descrito en la literatura científica la capacidad de $S$. marcescens de contaminar soluciones de clorhexidina acuosa y otros antisépticos (cloruro de benzalconio y triclosan), lo cual se atribuye a alteraciones en su membrana plasmática ${ }^{16,17}$.

Hasta donde sabemos, el nuestro es el primer reporte de contaminación intrínseca de clorhexidina por este microorganismo. En la búsqueda bibliográfica realizada, se encontró escaso número de brotes de $S$. marscesens asociado a antisépticos, correspondiendo todos a contaminación extrínseca. Asimismo, al revisar brotes por otros microorganismos que contaminan clorhexidina, en su mayoría corresponden a contaminación extrínseca. Destaca un reporte de contaminación intrínseca de jabón con clorhexidina al $2 \%$ por $K$. pneumoniae, en que los autores consideraron que posiblemente esta contaminación se debió a una concentración de clorhexidina menor a la rotulada, ya que los controles de esterilidad entregados por el fabricante estaban correctos. En nuestro caso, no existían controles de esterilidad, ya que no estaba normado la realización de estos controles en nuestro país ${ }^{9,10,15}$.

El brote contó con episodios de pseudo-bacteriemia, colonización, ISO e infección de sitio de inserción de diferentes tipos de catéteres, debido a que se usa clorhexidina acuosa en la preparación de la piel para obtener hemocultivos, además de la preparación pre-operatoria de la piel y curación de SIC y heridas. Si bien el uso de clorhexidina acuosa en la toma de hemocultivos no es la práctica habitual, este hecho nos permitió relacionar los casos y asociarlos a la fuente detectada.

Hubo una gran dispersión en el tipo de pacientes, tanto en edad como en diagnóstico de base, lo que en un principio dificultó el análisis, dado que no se encontraba factores de riesgo compartidos pero, precisamente ese hecho fue lo que nos indujo a buscar un elemento común entre todos ellos, que en este caso era el antiséptico utilizado.
Los pacientes infectados tuvieron una evolución variable, la mayor parte de ellos $(64 \%)$ requirieron reintervención, ya fuese para retiro de implante o aseo quirúrgico, y todos recibieron antimicrobianos. No hubo letalidad asociada.

Hubo cinco pacientes con cultivo positivo a $S$. marcescens en el período estudiado en los cuales no fue posible establecer el uso previo de clorhexidina, y correspondieron a muestras respiratorias (n: 4) y urinaria (n: 1). Se consideró que ellos no pertenecen al brote estudiado, posiblemente son parte de la endemia.

Se pudo demostrar la contaminación intrínseca del antiséptico, debido a que por norma en nuestra institución, los antisépticos utilizados vienen en presentación de $50 \mathrm{ml}$ y son de uso único, descartándose una vez abiertos, precisamente para evitar la contaminación extrínseca.

Se concluye en base al estudio molecular por EFCP que, tanto las ISO, ISC, las colonizaciones como las pseudo-bacteriemias, fueron producidas por la cepa encontrada en el lote de clorhexidina contaminado. Finalmente, el análisis precoz y alto grado de sospecha frente a la aparición de casos no habituales permitió descubrir de forma rápida, el origen del brote, con el consiguiente retiro del producto contaminado desde nuestra institución y luego en el país, evitando así la producción de nuevas infecciones.

Con posterioridad al estudio realizado, tuvimos acceso a un documento emitido por la empresa farmacéutica canadiense MEDISCA, alertando a nivel internacional la presencia de $S$. marcescens en materia prima de clorhexidina, fechado 14 de agosto de 2014, fecha anterior a la fecha de producción del lote de frascos contaminados detectado por nosotros.

Cabe la interrogante respecto de si se pudo evitar el brote de haberse tomado en consideración dicha alerta.

Esta es, sin duda, una oportunidad de mejora para el país, ya que a la fecha de este lamentable evento no era requisito certificar la esterilidad de los antisépticos, lo que claramente habría permitido evitarlo.

\section{Resumen}

Serratia marcescens es un bacilo gramnegativo de amplia distribución, frecuentemente asociado a infecciones nosocomiales. Se han descrito brotes asociados a la contaminación de diversas soluciones antisépticas. Describimos a continuación un brote de infección de sitio operatorio (ISO) y de infección de sitio de inserción de catéter vascular (ISC) por $S$. marcescens. A raíz de un caso índice se estudió un total de 33 pacientes con cultivo positivo para $S$. marcescens. El análisis epidemiológico, microbiológico y molecular logró demostrar la contaminación intrínseca 
de un lote de clorhexidina acuosa, como fuente común de exposición. Las muestras positivas correspondieron a 13 infecciones clínicas, nueve colonizaciones, seis pseudo-bacteriemias y cinco pacientes sin exposición demostrada. Los resultados de este estudio determinaron el retiro del producto de la institución y posteriormente a nivel nacional. La contaminación intrínseca de antisépticos es una causa poco frecuente de brotes de infecciones nosocomiales cuya identificación posee un gran impacto epidemiológico.

\section{Referencias bibliográficas}

1.- Mahlen S. Serratia infections: from military experiments to current practice. Clin Microb Rev 2011; 24: 755-91.

2.- Abbott S. Klebsiella, Enterobacter, Citrobacter, Serratia, Plesiomonas and other Enterobacteriaceae. Murray P, Baron E, Jorgensen J, Pfaller M, Yolken R, Manual of Clinical Microbiology 8th Edition, Washington DC, ASM press, 2003. P. 684-700.

3.- Dossi M, Escalona M, Serrano C, Silva M, Juliet C, Fernández A, et al. Serratia marcescens: descripción de un brote de infección intrahospitalaria. Rev Chilena Infectol 2002; 19: 262-6.

4.- Cano-González S, Romero-Vásquez A, Santamaría-Muñoz R. Factores de riesgo asociados a sepsis por Serratia marcescens en una unidad de cuidados intensivos neonatales. Salud en Tabasco 2004; 10: 214-20.

5.- Romano M L, Murguía P T, Pérez R V M, Santos P J I, Alcántar C D, Alpuche A C M. Brote de bacteremia nosocomial y colonización por Serratia marcescens en una unidad de cuidado intensivo neonatal. Bol Med Hosp Infant Mex 2007; 64: 9-15.
6.- Yoon H J, Choi J Y, Park Y S, Kim Ch O, Kim J M, Yong D E, et al. Outbreaks of Serratia marcescens bacteriuria in a neurosurgical intensive care unit of a tertiary care teaching hospital: A clinical, epidemiologic, and laboratory perspective. AJIC. 2005; 33: 595-601.

7.- Espinosa de los Monteros L E, Silva-Sánchez J, Jiménez L V, Rojas T, Garza-Ramos U, Valverde V. Ouitbreak of infection by extended-spectrum $\beta$-lactamase SHV-5 producing Serratia marcescens in a Mexican Hospital. J Chemother 2008; 20: 582-92.

8.- Okuda T, Endo N, Osada Y, Zen-Yoji H. Outbreak of nosocomial urinary tract infections caused by Serratia marcescens. J Clin Microbiol 1984; 20: 691-5.

9.- Weber D, Rutala W, Sickbert-Bennett E. Outbreaks associated with contaminated antiseptics and disinfectants. Antimicrob Agents Chemother 2007; 51: 4217-24.

10.- Chang CY, Furlong LA. Microbial stowaways in topical antiseptic products. N Engl J Med 2012; 367: 2170-3.

11.- Villarino M E, Jarvis W R, OHara C, Bresnahan J, Clark N. Epidemic of Serratia marcescens bacteremia in a cardiac intensive care unit. J Clin Microbiol 1989; 27: 2433-6.

12.- Vigeant P, Loo V G, Bertrand C, Dixon C, Hollis R, Pfaller M, et al. An outbreak of Serratia marcescens related to contaminated clorhexidine. Infect Control Hosp Epidemiol 1998; 19: 791-4.

13.- McAllister T A, Lucas C E, Mocan H, Lidell R H, Gibson B E, Hann I M, et al. Serratia marcescens outbreak in a pediatric oncology unit traced to contaminated chlorhexidine. Scot Med J 1989; 34: 525-8.

14.- Sánchez-Saldaña L, Sáenz-Anduaga E. Antisépticos y desinfectantes. Dermatología Peruana 2005; 15 (2): 82-103.

15.- Brooks S E, Walczak M A, Malcolm S, Rizwanullah H. Intrinsic Klebsiella pneumoniae contamination of liquid germicidal hand soap containing clorhexidine. Infect Control Hosp Epidemiol 2004; 25: 883-7.

16.- Lannigan R, Bryan L E. Decreased susceptibility of Serratia marcescens related to the inner membrane. J Antimicrob Chemother 1985; $15:$ 559-65.

17.- Marrie T J, Costerton J W. Prolonged survival of Serratia marcescens in chlorhexidine. Applied Environ Microbiol 1981; 42: 1093-102. 